\title{
Module Diagnostic-Training System-An Innovative Solution for Diagnosis and Assessment of Cognitive Processes
}

\author{
Magdalena Zubanska \\ Police Academy
}

\begin{abstract}
The development of cognitive competences for police officers and other officers of the uniformed services is crucial. The Police Academy in Szczytno (Poland) developed-as part of a research and development project (2013-2015) — an innovative diagnostic system for training that allows automated assessment of the current fitness level of cognitive functions and their online training. The system consists of three interconnected modules: diagnostic module, training module, and knowledge base. It is a fully functional online platform. Various forms of cognitive training in the form of games have been proposed. The system includes a component in the form of a psychophysiological recorder, which is intended for the training of coping with stress. The innovativeness of this diagnostic training system involves the use of information systems to stimulate the cognitive competence of officers by designing exercises in the form of computer games and to permit to verify their current mental shapes. It is assumed that this solution will develop the personal skills of officers and positively affect their operational readiness. Extending the area of application of this diagnostic training module by other functionalities can contribute to improving the effectiveness and safety of work, especially in occupational groups performing tasks requiring special cognitive and psychomotor predispositions.
\end{abstract}

Keywords: cognitive features, psycho-stimulator, diagnosis, training, uniformed services

\section{Introduction}

The concepts of public order and safety are inextricably linked with the functioning of the state. Ensuring public order is - in the light of human security - a key issue for the state. This task is performed by a number of entities that focus on the activities of supervising compliance with the law in the life of society simultaneously in many areas. In Poland, uniformed services are the bodies which are responsible for protecting the safety of people and property, for the maintenance of public order and prevention of crimes against human health and life. In this context, the Police is the organization of key importance for providing safety of state and society. In a dynamically changing environment, the management of organizations - including the organization of such a special meaning as the Police-requires constant improvement of the quality requirements for the management of the organization to be able to skilfully adapt to the needs of both external and internal expectations. A number of factors determine the efficiency of most organizations, but the key factor here is human capital. For this reason, the issue of the appropriate selection of officers for the implementation of the tasks arising from the legal provisions, needs, and statutory regulations is very important. It seems reasonable at this point to say that

Magdalena Zubanska, Ph.D., Institute for Research on Crime and Terrorism, Faculty of Internal Security, Police Academy, Poland; main research field: Forensic Science. 
the right person in the right place can ensure the effective implementation of the tasks assigned to them. It is worth emphasizing that the armed forces - especially the Police — are every day faced with tasks with a high degree of difficulty. It should be added that the work of a police officer is listed among the most aggravating occupations in the modern world. A number of factors affecting the level of mental workload of police officers are often mentioned. Generally, there is a distinction between factors connected with the on-duty and post-duty factors, although they often overlap, and these two spheres of activity influence each other. Uniformed services must continually improve their staff and pay attention to the right selection of officers to perform specific tasks assigned to them. In other words, we should aim to eliminate the randomness, because - as already mentioned - the effectiveness of these services depends to a very large extent on the human factor.

\section{Theoretical Rationale}

The quality of human functioning in various everyday situations depends on cognitive processes that are used to learn about the environment. Perception, attention, memory, thinking, decision-making skills, as well as emotional features, are cognitive processes whose reliability is essential. They fulfil an important adaptive function. Thanks to them, a human being has the flexibility to adapt to constantly changing conditions. In addition, these processes increase the chances of the correct response (Bonus-Dziego 2015, 91). The effectiveness of uniformed officers is conditioned by many factors, yet the particular importance is attributed to the area of cognitive competence. Developing the necessary cognitive functions by a uniformed officer is a response to new challenges, posed by constantly dynamically changing situations. Moreover, the development and improvement of cognitive skills is one way of obtaining higher efficiency in the implementation of official tasks entrusted to officers. To meet the needs in the development of cognitive skills, the Police Academy in Szczytno (Poland) developed the concept of a research and development project entitled Development of Interactive Training Psycho-Stimulator for the Police (No. 3/OROB 000503 001/13). The project proposal was submitted in 2012 in the competition for the execution of projects in the field of research and development for defence and security announced by the National Centre for Research and Development (NCRD). It is worth noting that the National Centre for Research and Development (established in Warsaw, Poland) is an executive agency of the Minister of Science and Higher Education. It is a unit implementing the tasks of science policy, technology, and innovation. The Centre provides a platform for effective dialogue between science and business environment. The Centre is funded by the State Treasury and the European Union funds.

The project received funding from NCRD and in December 2012 the project works began. Implementation of the project ended in 2015. The aim of the project was to develop and implement an innovative diagnostic and training system to evaluate and stimulate cognitive competences of officers. The legitimacy of the development of innovative tools to support activities aimed at developing the cognitive potential of officers of the uniformed services was confirmed during research phase. As a result of exploratory studies, such cognitive processes as memory, perception, attention, and logical thinking were considered as the most important factors for the effective and efficient work of police officers (Zubanska et al. 2014, 157).

\section{Structure of the Diagnostic and Training System Module}

The result of the completed project is an innovative tool in the form of a modular diagnostic and training system to evaluate and stimulate cognitive competence. The system consists of three interconnected modules: diagnostic, training, and the library module (knowledge base). The system further includes a recorder designed 
for psychophysiological training to cope with stress. Each stage of the project includes a study standardization, optimization, and validation of the system.

\subsection{Diagnostic Module}

Diagnostic module consists of four components. Each component is a set of psychological tests to allow independent measurement of the level of the following cognitive skills: concentration, observation, working memory, and logical thinking. To assess the capacity of each of these skills, a separate test is developed. The module allows for automatic assessment of the current fitness level of the cognitive functions of individuals to determine the level of output and the zone of proximal development. The tests available to assess these cognitive functions are fully functional psychological tests with proven psychometric properties (standard norms were calculated for each test separately); as the standard scale we used percentile scale. In the construction of individual tests, we sought to ensure the attractiveness of the procedures that were not tedious for the user (Gęga 2015, 122).

\subsection{Training Module}

Training module is linked to a set of games to stimulate cognitive competence, allowing their training online using both desktop PCs, mobile smart phones, or tablets. Introduction of the system on the Internet platform allowed users to obtain feedback on their progress in training in real time, as well as compete with each other. It was assumed that the training system through games should include material that was attractive thematically and formally for the officers as much as possible. It was assumed that the game would refer to the actions often undertaken by police officers (perception, memory, describing scenes and characters, deduction, combining the facts or the fight or chase/pursuit). At the same time, a player's task should require periodic full engagement of processes such as attention and memory. Making the right decisions in stressful situations is associated with far-reaching control of not only the emotions but also reflexes. As shown by scientific data and observation practices of police officers, such control (if it is part of the daily experience of officers in recurring work situations for a long time) can lead to accumulation of negative effects of experiencing stress (loss of control in situations neutral both at work and during free time) as well as professional burnout and psychosomatic disorders. For this reason, an important part of the game is to construct a training situation requiring intensive control of impulsive and emotional reactions. Tasks requiring perception enforce not only the perception of certain objects, but also their perception according to established rules. Some tasks require to restore the likely sequence of events. Tasks requiring the use of memory were focused on the storage of visual as well as verbal information. Higher level tasks involve capturing the logical relationships between objects (e.g., the need to memorize a series of characters that have one thing in common). Tasks that require continuity and selectivity of attention consist of tracking of certain elements among many distractors. The tasks are diverse in terms of the strength of stimulation. These are both tasks that require working under strong pressure, as well as monotonous tasks, i.e., tasks requiring the maintenance of vigilance despite the relatively weak stimulation. The subject of each game/task is connected to a common storyline (which refers to a single universum).

Managing the level of tasks difficulty means optimizing the selection of tasks for the current efficiency of the training. It is based on the principle of the zone of proximal development, so that the person receiving training tasks which they are not able to perform for the first time, but whose level of difficulty guarantees the possibility of success after several attempts. It is also important to include the element of joy, i.e., the trainee 
should feel genuine satisfaction from winning and self-development. Each of the basic levels of the game is designed to stimulate one selected function/skill as much as possible, without involving others. Subsequent levels bring hindrance. At some point, in each game, the trainee is allowed to practice two or more functions. At this point, the difficulty of the exercising basic function is maintained unchanged and additional function is exercised beginning from the lowest level. The condition that must be met to unlock the level of practicing two functions simultaneously is that the trainee must pass the test from each of them separately at least at the basic level. It is important to design the system in which the last level in each game is open, and therefore contains an algorithm to automatically increase the level of difficulty with no pre-set limit. This will help maintain the motivation of even advanced users of the system. To sum up, the tasks of training are developed in the form of computer games based on advanced algorithms for determining the difficulty of the various aspects of the game. A total of eight training versions are developed dedicated for training four separate cognitive functions. Subsequent games are designed as exercises with increasing difficulty. The first four training sessions are focused on the development of individual functions singled out as crucial (see above). The other four are sets of exercises, during which one of the main cognitive functions is trained with elements engaging other functions. Such gradation of difficulty helped to balance the whole training programme and provide training close to real conditions (Szostok 2015, 127).

The entire procedure using the diagnostic training system runs according to the scheme: diagnosis-training-diagnosis. The first step is a preliminary study of a separate skill, i.e., memory, attention, perception, and reasoning to determine the level of output and the zone of proximal development. After these preliminary studies, a person is redirected from the diagnostic module for the training module, on which a game taking into account the current level of cognitive ability is activated. After a specified time of training, the participant returns to the diagnostic module for another test allowing verification of the effectiveness of training in improving cognitive processes, which enables the measurement of progress (Gęga 2015, 125).

\subsection{Library Module}

The library module contains a collection of information and studies on issues relating to the development of cognitive functions. Materials are grouped thematically into four virtual shelves, which are named: logical thinking, memory, perception, concentration and attention.

\subsection{Psychophysiological Recorder. PST Training}

The diagnostic training system includes a module in the form of psychophysiological recorder, whose task is to expand the training of elements of learning to cope with stress. It is a device to evaluate the intensity changes of (selected) psychophysiological reactions integrated with games and tests and special software. It should be noted that the fact of self-awareness of tension and physiological changes, which are affected by performing difficult tasks, is really important, being the basis for the evolution in the way of response, e.g., in critical situations. Before the task, the purpose of which was to develop this psychophysiological recorder, we had analysed such problem areas as exposure to stress in the police, psychophysiological reactions to stress, exposure to acute and chronic stress, suppression of emotions as a source of occupational stress, self-preservation as a source of occupational stress, coping with destructive effects of stress, stress reduction, biofeedback, "inoculation training" and stress-related disorders. 
The psychophysiological recorder is a module for measurement, which gives a person the ability to control the level of the measured variables. This makes it possible to obtain knowledge of the reactions, of which we are very often unaware but which have a significant impact on the actions taken. The module is developed as an independent portable device that communicates using a SPP protocol (Serial Port Profile) via Bluetooth and is adapted to work indoors. The recorder is characterized by, among others, the fact that it is compact, portable and has a band for attaching it to the wrist of the arm. At this point, it is worth mentioning that the work on the concept of the psychophysiological recorder module has been inspired by solutions used in a polygraph device (Zubanska 2014, 137). Modern polygraphs are devices that reveal and record the current level of emotional activation based on changes in respiration, skin conductance, and changes in pressure and pulse rate. Data recording is done by sensors attached to the person's body, and the changes are saved in the form of charts, separately for each run (channel). In the currently used polygraphs, the data are recorded with the following sensors:

(1) Breathing patterns are recorded by pneumographs;

(2) Using GSR sensors (Galvanic Skin Response), the ectodermal activity is recorded reflecting the relative change in conductivity or current and resistance in the epidermis tissue;

(3) Relative changes in the rate and pulse amplitude and relative blood volume are recorded by a cardiograph (Gołaszewski 2013).

Psychophysiological recorder is equipped with a sensor recording pulse and a sensor to measure the level of skin-galvanic response (electrodes to measure changes in the intensity of galvanic skin-response, GSR).

Using the psychophysiological recorder module is about the fact that the user can connect himself/herself to simple sensors attached to the fingers of the left hand (or right hand if he/she is left-handed) and then perform the tasks in the game (using the training module) requiring rapid action and high reflexes. It should be mentioned that some of the tasks are performed in the considerable pressure of time and with the additional aversive stimuli (e.g., sudden, unpleasant sound in headphones), which may in some people result in a tendency to act in a hurry, with increased levels of stress and, consequently, result in inefficient execution of the task. The system then reads the user's psycho-physiological parameters and after the game it provides feedback describing the changes in the reactions of the body, from which we can assess the level of stress in the person. After performing several tasks, the user can assess the effects of their training by analysing and comparing the records done by the psychophysiological recorder for each training session. Summing up, we are dealing with a device for the assessment of changes in the intensity of (selected) psychophysiological reactions integrated into the system of games and software for analysis of results. Psychophysiological recorder can be regarded as a helpful tool to raise awareness about physiological responses of trained persons in a variety of test conditions and the games that are intended to substitute for the real situations and tasks in which police officers may find themselves.

It should be noted that the evaluation of recorded parameters is not possible to be done "live" during training (i.e., in the course of the game) and only after its completion. It is expected that persons actively training should focus on the task rather than on emotions. Interpretation of results will then enable practicing individuals to develop effective ways of coping with stressful situations. Now it can be said that the most authoritative and clear indicator of fluctuations in the emotional state of the user record will be the registered galvanic skin-response (GSR channel). 
For polygraph studies, which constituted a reference point here, it is the measurement of skin conductance (GSR) that is for diagnostic purposes particularly important (Handler et al. 2010). According to the latest empirical data, the GSR channel is of greatest contribution to the evaluation of the test results (which uses polygraph)-indicated in 53\%.

The task of the psychophysiological recorder is to support work on the control of emotions by presenting feedback on psychophysiological arousal level during the implementation of elements of the training characterized by strong pressure. The method of offline psychophysiological analysis is developed for the training, which is called PST training. Its essence lies in the recording psychophysiological parameters during the execution of computer exercises that can cause negative emotions and stress (acting under the pressure of time, frustration, etc.). After completing the assumed set of exercises, the system calculates the parameters and displays information about the level of psychophysiological arousal during periods designated by markers of time associated with the executed task. The training person (using the training module) can again do the task with similar characteristics and in this way get feedback on the changes of parameters in order to learn optimal strategies to control emotions.

In a stressful situation, we distinguish the evaluation of the primary phase (increased arousal in a situation in which the body needs to do more work) and evaluation of the secondary phase (the individual assesses the opportunities and risks and chooses the optimal strategy). During this phase, the right style of concentration is developed, i.e., either on the task, on their own emotions, or on avoiding stress. Depending on what is the purpose of training, the user can choose the right style, i.e., biofeedback or offline technique. Just to remind, the biofeedback method involves immediate presentation of psychophysiological parameters of a person doing training, so that they are able to systematically control their level of stimulation and work out suitable relaxation techniques. The main difference in the application of both methods is reduced to the entire workout. If the purpose of the training is to improve insight, understanding of their own emotions and guide them during the execution of a task, we use the method of biofeedback. However, focusing on emotions and controlling them, watching the indicators which display online values of individual aspects of arousal is difficult to simultaneously focus on the tasks to be performed. Therefore, in a situation where it is essential to concentrate on the performed task and to control emotions, it is advisable to use the offline technique. It allows a person to focus attention on the task. Assessing the emotional "costs" and learning control over them takes place during the debriefing which also includes the assessment of performance of the training.

All in all, biofeedback proves to be a useful technique if the purpose of the training is to strengthen the style focused on emotions (which is sometimes important, however, it is demonstrated that the police officers who focus on emotions make more mistakes and are less able to cope with the performance of duties). Very often there is a need to develop the working style focused on the task, so for this purpose the method of recording and offline analysis using the psychophysiological recorder module is recommended. The results are recorded by the device and then analysed offline (i.e., after the task is completed) in order to evaluate progress in reducing psychological stress. The developed PST training system is based on the principles of gamification (Szostok 2015).

\section{Conclusions}

In modern times, professional competences coexist and interact with the dynamic development of scientific thought and technological progress. Skills relying on particular ways of thinking, as well as information 
processing, synthesis, and analysis of various data have become more important (Nosal 1999). This fact will most likely determine the growth of interest in aspects of intellectual capacity. The empirical data suggest that the level of intellectual capacity is one of the best predictors among others in many areas of social life, as for instance success in professional career (Sękowski 2006). Project assumptions about the legitimacy of development and improvement of cognitive skills as a way to obtain higher efficiency in the implementation of tasks assigned to officers of the uniformed services (including police officers) are fully reflected in the triarchic theory of intelligence developed by R. J. Sternberg, according to which intellectual abilities can be developed and improved. Thanks to this, in turn, human beings can more effectively achieve goals and perform professional tasks assigned to them (Gęga 2015).

Legitimacy to develop awareness of the impact on the effectiveness of performed cognitive tasks is unquestionable. From a psychological point of view, the innovation aspect of the project lies in the precise identification of cognitive and intellectual functions, which are essential in the work of the uniformed officers and developing methods to stimulate these functions through proper training. It is assumed that the implementation of the outcomes of the project should contribute to improving the quality of personnel, improving their self-esteem and mental efficiency, and thereby increasing the potential of these services. The security of the state and its citizens is strongly influenced by efficient law enforcement authorities. Therefore, appropriately matched and trained officers, who have insight into their potential and can maintain cognitive functions at the highest possible level (through adequate training of the mind), are a guarantee of good and an effective implementation of tasks.

\section{Works Cited}

Zubanska, Magdalena, Bonus-Dziego Agnieszka, and Zubrzycka Anna K. Training Interactive Psycho-Stimulator for the Police. Ed. Pracana Clara. Psychology Applications \& Developments. Lisboa: inScience Press, 2014.

Bonus-Dzięgo, Agnieszka. Characteristics of Selected Cognitive Processes. Ed. Ścibiorek Zbigniew. Diagnosing Abilities of Representatives of Uniformed Services. Szczytno: Police Academy in Szczytno, 2015.

Gołaszewski, Marcin. Current Standards of Polygraphic Research. Warszawa: Internal Security Agency, Central Training Center, 2013.

Zubańska, Magdalena, Bonus-Dzięgo Agnieszka. "Psycho-Physiological Recorder-Seeking Inspiration from the Polygraph." Internal Security (July-December) 6.2 (2014): 135-51.

Szostok, Krzysztof. Mechanisms of Gamification as an Element of Supporting Programs of Cognitive Processes' Development of Uniformed Services Officers. Ed. Ścibiorek Zbigniew. Diagnosing Abilities of Representatives of Uniformed Services. Szczytno: Police Academy in Szczytno, 2015.

Gęga, Monika. Cognitive Potential-Its Role in Improvement of Work Efficiency in Uniformed Services. Ed. Ścibiorek Zbigniew. Diagnosing Abilities of Representatives of Uniformed Services. Szczytno: Wyższa Szkoła Policji w Szczytnie, 2015.

Handler, Mark, Nelson Raymond, Krapohl Donald, and Honts Charles. "An EDA Primer for Polygraph Examiners.” Polygraph 2.39 (2010): 68.

Nosal, Czesław. Psychology of Personnel Decisions. Kraków: Press of Professional Business School, 1999.

Sękowski, Andrzej. "'Stimulation of Abilities' Development in the Light of Robert J. Sternberg's Theory of Successful Intelligence.” Psychological Yearbooks IX.1 (2006). 